\title{
Thermodynamic Efficiency Maximum of Simple Organic Rankine Cycles
}

\author{
Aram Mohammed Ahmed ${ }^{1,2}$ (D) László Kondor ${ }^{1}$ and Attila R. Imre ${ }^{1,3, *}$ \\ 1 Department of Energy Engineering, Faculty of Mechanical Engineering, Budapest University of Technology \\ and Economics, Múegyetem rkp. 3, H-1111 Budapest, Hungary; arammohammed@energia.bme.hu (A.M.A.); \\ kondorlaszlo94@gmail.com (L.K.) \\ 2 Technical College of Kirkuk, Northern Technical University, Kirkuk 36001, Iraq; aram.mohammed@ntu.edu.iq \\ 3 Centre for Energy Research, Department of Thermohydraulics, POB. 49, H-1525 Budapest, Hungary \\ * Correspondence: imreattila@energia.bme.hu or imre.attila@ek-cer.hu
}

Citation: Ahmed, A.M.; Kondor, L.; Imre, A.R. Thermodynamic Efficiency Maximum of Simple Organic Rankine Cycles. Energies 2021, 14, 307. https://doi.org/10.3390/en14020307

Received: 18 December 2020 Accepted: 5 January 2021 Published: 8 January 2021

Publisher's Note: MDPI stays neutral with regard to jurisdictional clai$\mathrm{ms}$ in published maps and institutional affiliations.

Copyright: (C) 2021 by the authors. Licensee MDPI, Basel, Switzerland. This article is an open access article distributed under the terms and conditions of the Creative Commons Attribution (CC BY) license (https:// creativecommons.org/licenses/by/ $4.0 /)$.

\begin{abstract}
The increase of the maximal cycle temperature is considered as one of the best tools to increase cycle efficiency for all thermodynamic cycles, including Organic Rankine Cycles (ORC). Technically, this can be done in various ways, but probably the best solution is the use of hybrid systems, i.e., using an added high-temperature heat source to the existing low-temperature heat source. Obviously, this kind of improvement has technical difficulties and added costs; therefore, the increase of efficiency by increasing the maximal temperature sometimes has technical and/or financial limits. In this paper, we would like to show that for an ideal, simple-layout ORC system, a thermodynamic efficiency-maximum can also exist. It means that for several working fluids, the thermodynamic efficiency vs. maximal cycle temperature function has a maximum, located in the sub-critical temperature range. A proof will be given by comparing ORC efficiencies with TFC (Trilateral Flash Cycle) efficiencies; for wet working fluids, further theoretical evidence can be given. The group of working fluids with this kind of maximum will be defined. Generalization for normal (steam) Rankine cycles and $\mathrm{CO}_{2}$ subcritical Rankine cycles will also be shown. Based on these results, one can conclude that the increase of the maximal cycle temperature is not always a useful tool for efficiency-increase; this result can be especially important for hybrid systems.
\end{abstract}

Keywords: ORC; Trilateral Flash Cycle; T-s diagram; adiabatic expansion; working fluid; retrofit; $\mathrm{CO}_{2}$ power cycle

\section{Introduction}

Organic Rankine cycle-based processes are popular and effective methods to utilize heat sources with a wide range of temperature to utilize for electricity production. Most of the heat sources (like geothermal heat, industrial heat) are localized. Sometimes, there is a possibility to use an additional, non-localized heat source (like solar heat) to increase the maximal cycle temperature. For the basic thermodynamic cycle (Carnot-cycle), the first law efficiency is increasing by increasing the maximal cycle temperature while minimal cycle temperature is constant [1]. For other cycles, a similar law can be used, only in that case, maximal and minimal cycle temperatures have to be replaced by mean temperatures of heat addition and removal [2]. Using the assumption, that mean temperature of heat addition is increased when maximal cycle temperature is increased [2], a thumb-rule can be deducted, that by increasing the maximal cycle temperature, the cycle efficiency is always increased. Therefore, the increase of the temperature is always desirable in simple thermodynamic cycles, when the goal is the better performance, although other constraints (like the cost of the utilization of this added heal source) can overshadow the gain caused by the efficiency increase.

The design of an ORC-based power plant has at least four different layers [3]. The first one is about the thermodynamic cycle itself; one can design and optimize the Rankine- 
cycle using various working fluids and various constraints (like defining the "ideality" or "reality" of the cycle by defining the irreversibility-induced entropy-production during expansion). The second layer is the technical/engineering layer; the designed Rankinecycle should be realized, the constraints of the previous steps have to be associated with various hardware components or process properties (like expander internal efficiency, pressure loss within the heat exchanger, etc.). An optimal second layer design might require several successive approximation steps, where the first step design has to be recalculate due to various points (for example inaccessibility of expander with the desired internal efficiency), and the new result has to be used for the next iteration. The third layer is the economical one. One should realize that investors built power plants not to generate electricity but to generate profit. Therefore, even the most brilliant thermodynamic cycle or ORC layout might be rejected upon financial points. The fourth layer is the environmental one; it is strongly connected to the third one [4].

With a bit of oversimplification, one can say that in the first layer, the thermodynamic properties of the working fluids, especially the shape of their $T$-s diagram are the most important factor [5,6], involving even molecular properties [7,8], while in the second layer already having the working fluid, the proper choice of technological components (most often the expander) plays the leading role $[9,10]$. However, sometimes these two steps are very much interlocked [11]. Obviously, the final vote is always for the economic side [4].

To have successful optimization in the second layer, one should clarify the problems raised in the first layer. Although most people assume that thermodynamics already solved all related problem and no further study is necessary concerning basic cycles, it is not a valid assumption. Here, we are going to show a clear example to disprove this assumption.

ORCs are supposed to be used to utilize heat sources not utilizable with traditional steam Rankine cycle; these heat sources are most often low-enthalpy ones with relatively low temperature and sometimes with low heat flow. For this reason, ORC-based power plants have limited financial viability; investment, operational and maintenance cost should be kept as low as possible. One of the ways to do that is the use of the basic ORC layout, namely one with a heater (liquid heater plus evaporator), an expander, a condenser and a pump, without using superheater or regenerative/recuperative heat exchanges [12]. Addition of any extra component can increase investment cost and decrease the "robustness" of the design. Therefore, our goal is to solve the thermodynamic problems without the involvement of any new part, i.e., using only the basic ORC design.

In this paper, we would like to show, that using the simplest ORC (or even traditional, water/steam-based RC) layout, a thermodynamic efficiency maximum should be found in all ideal cycles using wet and in several ideal cycles using dry working fluids. To prove this statement, the efficiencies of simple ideal ORC and TFC (Trilateral Flash Cycles) processes will be studied, using working fluids from various classes. The exact location of these maxima depends on the working fluid, as well as on the minimal cycle temperature. The existence of this kind of maximum shows that the increase of the maximum cycle temperature is not always a proper tool to increase cycle efficiency; sometimes it can be contra-productive.

\section{Hybrid Systems}

The temperature of the heat source is an important factor for ORC applications, even though this technology can utilize sources with relatively low temperature. Geothermal energy is often considered as a low-grade energy-source; therefore, it cannot independently support high load applications. This is true even for countries with quite good geothermal potentials (like Hungary), where the well-head temperature of most of the existing geothermal wells are below $90{ }^{\circ} \mathrm{C}$ [13]. These kinds of sources are usually excluded from the pool of potential sources for electricity generation, although they can be numerous and some of them have very impressive heat-flux. In this case, one might apply hybrid systems (using secondary heat sources with a smaller heat flux but with higher temperature) to overcome the inherent weakness of the low-temperature sources. 
Probably the most frequently used double-source design is the hybrid solar-geothermal installation [14-16]. For interested readers, a short overview of these kinds of systems is given in Appendix A. In hybrid solar-geothermal systems, the initial heating can be done by the low-temperature geothermal source, and then the solar heat is used to increase the maximal temperature. This can be done in two different ways. In the first solution, the solar heat can be used to "superheat" the already evaporated vapor [17]; this solution can be easily applied in retrofitted systems because only minor modifications of the existing geothermal power plant are required. In the second solution, the geothermal heat is used to preheat the compressed liquid, while the solar heat would be used to reach the maximal temperature and for evaporation. In this case, the pressure in the evaporator, as well as the input pressure of the expander, will be higher than for the same systems without solar heat; therefore, this method is not ideal for retrofitting of existing systems, but might be better for novel units [18]. The choice between the two options depends not only on the temperature values of the two sources but also on the available heat flows; in case of very small secondary heat flow, only the first case could be a plausible choice. Here, we prefer the second method, because our simple layouts would not consist of superheating units, i.e., the ORC design would remain simple.

Maximal cycle temperature, which is an important factor of the efficiency, can be increased in three ways in solar or hybrid solar systems:

- It is possible to keep the original solar collectors (i.e., total heat flux and heat source temperature remain the same), while the mass flow of the working fluid could be reduced. A small increase in the maximal cycle temperature can be obtained, but the simultaneous decrease of the pinch temperature (the minimal temperature gap in the heat exchanger) does not allow the significant increase. In this case, the investment cost of the retrofit would be minimal or zero, but the decrease of mass flow might decrease the power; therefore, relative (power-normalized) investment cost can increase.

- The number of solar collectors can be increased, increasing the collected heat but keeping the source temperature. In this way, one can keep the mass-flow of the working fluid, but pinch temperature can be slightly decreased while the maximal cycle temperature can be increased (only on a small extent). This solution, due to the extra collectors, increases the investment cost.

- $\quad$ The quality of solar collector system can also be improved (for example by changing the simple flat collectors to a focused system). With this solution, not only the amount of collected heat but also the temperature can be higher. In this way, because the source temperature is higher, keeping the mass-flow, the maximal cycle temperature can be remarkably increased. This solution, due to the use of better, i.e., more expensive collectors increases the investment cost.

One might expect, that just like for the ideal Carnot-cycle [1]; increasing the maximal cycle temperature for ORC or similar cycles would increase the thermal efficiency at least up to the critical point and therefore the application of an additional heat source would be limited only by technical or economic constraints [3]. Here, we are going to show that by using a basic ORC cycle, thermodynamic efficiency can have a maximum, associated with a sub-critical temperature, which depends on the material and the minimal cycle temperature.

\section{Method}

Concerning the basic ORC system, we are using the most straightforward and simplest layout to minimize the installation and maintenance costs for the system. The layout assuming a hypothetical dry working fluid can be seen in Figure 1a. It contains a pump, used to compress the working fluid from the low-pressure liquid state (1) to high pressure one (2). The first heat exchanger is for heating the compressed working fluid. It has two parts, the liquid heater ( $\mathrm{LH})$ to heat up the compressed liquid to the saturated state $(2 \rightarrow 3)$ and the evaporator $(\mathrm{EV})$, to evaporate the saturated liquid to saturated vapor state $(3 \rightarrow 4)$. Cost minimization comes here first; no superheater is included, not even for wet working 
fluids. The fluid expands directly from this saturated vapor state (4) through the expander (E) to cold, dry vapor state (5). It will be cooled down in the condenser (C) in two steps, first by pre-cooling the vapor down to the saturated state $(5 \rightarrow 6)$, then by condensing it to the saturated liquid state (1). The heat extracted by the pre-cooler is lost here; for the sake of simplicity, recuperative or regenerative heat exchangers are omitted. Corresponding $T$-s diagram can be seen in Figure $1 \mathrm{~b}$. Using wet working fluid, the expansion line $(4 \rightarrow 5)$ would run in the two-phase region (wet vapor) and points 5 and 6 would be identical; therefore, pre-cooling would not be necessary.

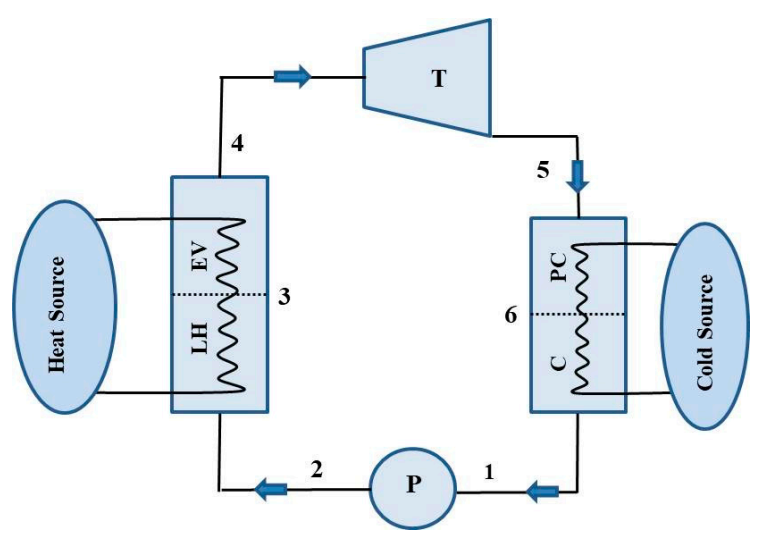

(a)

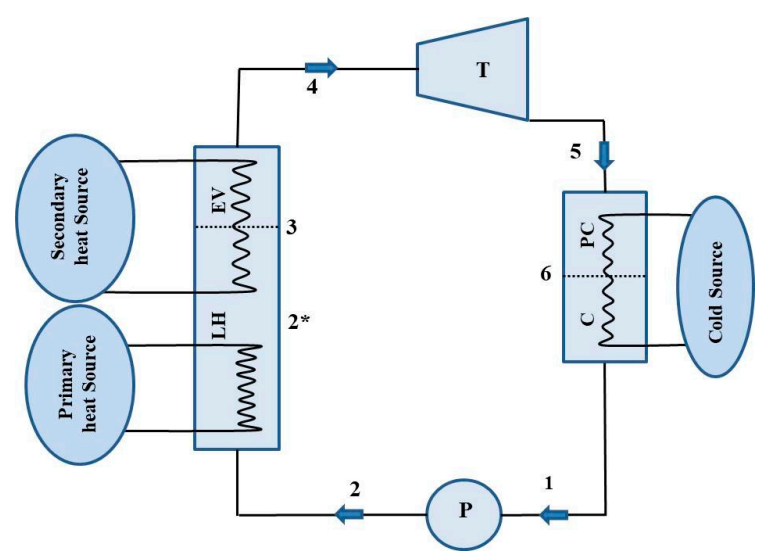

(c)

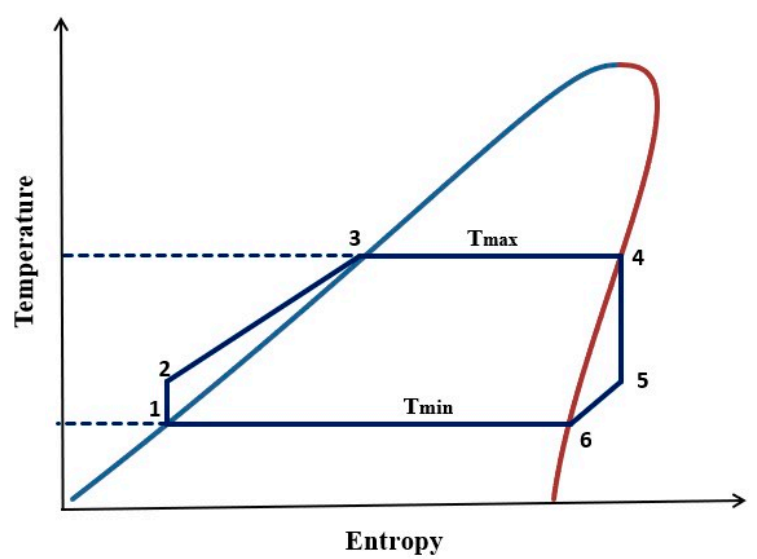

(b)

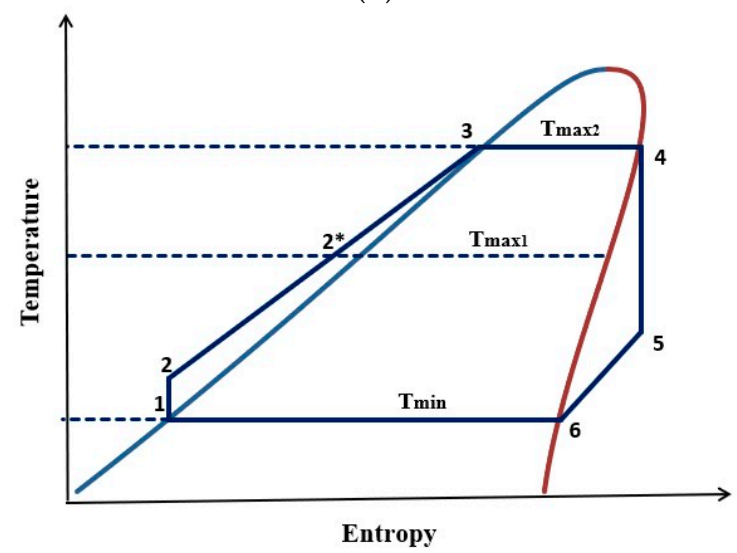

(d)

Figure 1. Schematic layouts and T-s diagrams for simple single-source (a,b) and hybrid (c,d) ORC systems. P: pump; LH: liquid heater; EV: evaporator; T: turbine or expander; PC: pre-cooler; C: condenser.

For the hybrid system, we have a second heat source; corresponding figures are Figure 1c,d. In this case, higher initial compression (from point 1 to 2 ) is used, so the pressure ratio $p_{2} / p_{1}$ will be higher. Pressurized working fluid (in liquid state) will be preheated by the primary heat source of the maximal temperature provided by the primary source (from point 2 to point $2^{*}$; this was the point corresponding to maximal temperature in the single source cycle). Then the secondary heat source (for example the solar one) is heating the fluid up to the maximal cycle temperature (from point $2^{*}$ to point 3 ), and also the heat of this source is used to evaporate it (from point 3 to 4). Expansion and cooling steps (the 4-5-6-1 sequence) would be similar to the previous case, although the expansion part (from point 4 to 5) will be more significant and the cooling of the dry vapor (5-6) would start at a higher temperature.

As it has been already stated, we are discussing only the thermodynamic cycle here; therefore, all steps are considered to be ideal. Compression and expansion steps are isentropic, while for the heating and cooling steps, pressure drops are omitted, and strict 
isobaric heat exchange processes are assumed. Because for first-law efficiency, the knowledge of the intermediate temperature $\left(T_{\max 1}\right)$ is not needed, only the effect maximal cycle temperature $\left(T_{\max 2}\right)$ will be investigated.

Although wet working fluids are considered as not suitable ones for ORC applications [19], due to their unfavorable behavior (most remarkably the ones related to the appearance of droplets), in this paper we are studying the thermodynamic, rather than technical aspects of ORC. Therefore, both dry and wet working fluids were checked.

Efficiencies are going to be calculated for six different materials (see Table 1), representing a wide range of working fluids from wet ones (almost symmetrical, bell-shaped T-s diagrams) to very dry ones (with very skewed T-s diagrams), see Figure 2. Four of them might be used for hybrid geothermal/solar ORC or RC while the other two having very low critical temperature might be used in cryogenic cycles [20-22]. Therefore here-when the heat source is solar heat, presumably providing source temperatures exceeding the critical temperatures of these two materials-they are only used for demonstration purpose. Two of them are wet working fluids (using the traditional classification), or class ACZ working fluids [23]. The other four are dry working fluids. Concerning the sequence-based classification [23], dry working fluids can be divided into two classes; ACZM and AZCM types. Letters represent the special point on the T-s diagrams; $A$ is the initial point of the T-s saturation curve, $\mathrm{Z}$ is the final one, $\mathrm{C}$ is the critical point and $\mathrm{M}$ is the point corresponding to maximal entropy. From these points, four-letter sequences can be built, based on the entropy values of these points. Initial point $\mathrm{A}$ always has the smallest entropy, while $\mathrm{M}$ has the highest one; therefore, only sequences starting with $\mathrm{A}$ and ending with $\mathrm{M}$ are possible. Having only two intermediate points, these are the ACZM and AMCZ. Liquid states are located in the A-C part of the curve, while vapor ones are stretching in the C-M-Z part. The crucial difference is that for ACZM, the entropy values for liquid states are always below the entropies of any of the saturated vapor states; while for AZCM, there are liquid states with entropies higher than for some vapor states. This means that for AZCM-type fluids, isentropic expansion starting from some saturated liquid states can reach the fully vaporized dry-vapor region. The ACZM-ones are closer to the wet-ones (ACZ), as it is shown in Ref. [21]. Three of the working fluids represented here are ACZM, and one for AZCM. In several fluids, a local entropy minimum $(\mathrm{N})$ also can be seen; these fluids can also be classified as dry ones, or alternatively as "real isentropic ones" because, for them, it is possible to have an isentropic expansion step from saturated vapor state into another saturated vapor state $[21,23,24]$. In this paper, this fifth point will not be relevant.

Table 1. Working fluids used in this study with some of their relevant properties.

\begin{tabular}{ccccc}
\hline Name & Type & $\begin{array}{c}\text { Class } \\
\left.\text { (Absolute/at 20 }{ }^{\circ} \mathbf{C}\right)\end{array}$ & $\boldsymbol{T}_{\text {cr }}[\mathbf{K}]$ & $p_{\text {cr }}[\mathbf{M P a}]$ \\
\hline Water & Wet & ACZ/ACZ & 647.1 & 22.06 \\
Carbon dioxide & Wet & ACZ/ACZ & 304.13 & 7.38 \\
Hexafluoroethane (R116) & isentropic & ACNMZ/ACZ & 293.03 & 3.05 \\
Butane & Dry & ACNMZ/ACZM & 425.12 & 3.8 \\
Neopentane & Dry & AZCM/AZCM & 433.74 & 3.2 \\
Dodecane & Dry & AZCM/AZCM & 658.09 & 1.82 \\
\hline
\end{tabular}

One more piece of information is needed concerning the use of this working fluid classification [23]. The ultimate initial and final points of the saturation curve in T-s diagrams are related to the triple points (the last point of the liquid/vapor equilibrium; solid-phase appears at that point). The corresponding entropy values are material properties, and therefore the classification (being the location of $\mathrm{C}$ and $\mathrm{M}$ points also material-dependent) is also a material property. However, in several cases, triple points are located in technically irrelevant, low temperatures (like for butane, where it is located at $134.6 \mathrm{~K}$, which is $-138.5^{\circ} \mathrm{C}$ ). Therefore, it might be better to terminate the T-s curve in a technologically more relevant minimal temperature, for example, at the minimum cycle temperature. In ORC applications 
(with air cooling) this should be done somewhere around (or exactly at) $20^{\circ} \mathrm{C}(293.15 \mathrm{~K})$. The relevant part of the curve (relevant means that the one above $20^{\circ} \mathrm{C}$ ) might mimic a novel class; for example, the vicinity of the top of all the T-s diagrams (above point $\mathrm{M}$ ) looks like a wet, ACZ type curve. For the sake of clarity, sometimes these "relative" classes are distinguished by marking the related points with an upper-case star $\left(^{*}\right)$, like $\mathrm{A}^{*} \mathrm{CZ}{ }^{*} \mathrm{M}$. Since for hybrid solar/geothermal case, usually, $20^{\circ} \mathrm{C}$ can be taken as minimum temperature; therefore, both absolute and relative classes are shown in Table 1.

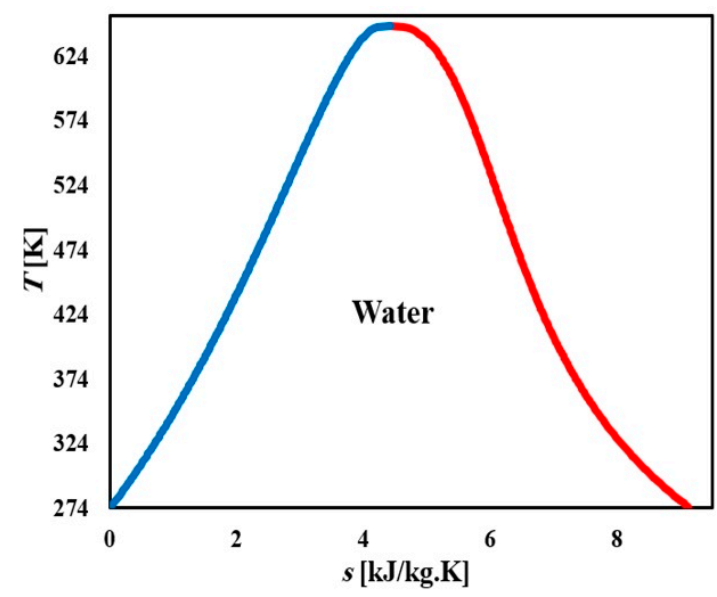

(a)

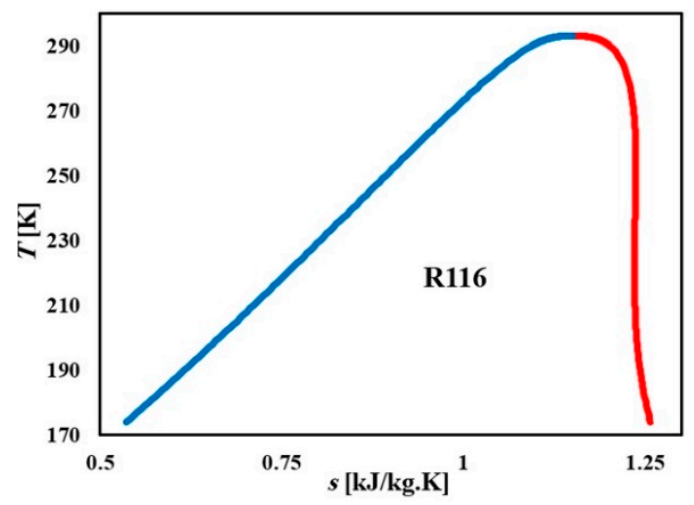

(c)

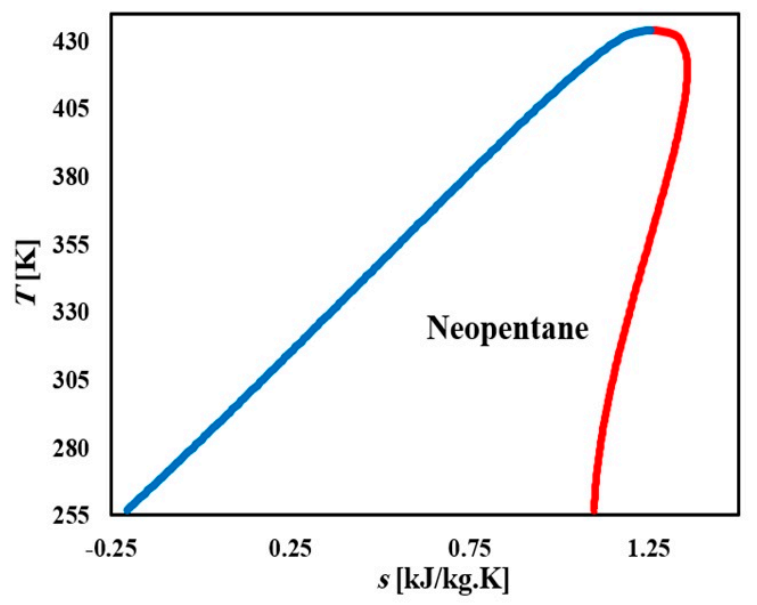

(e)

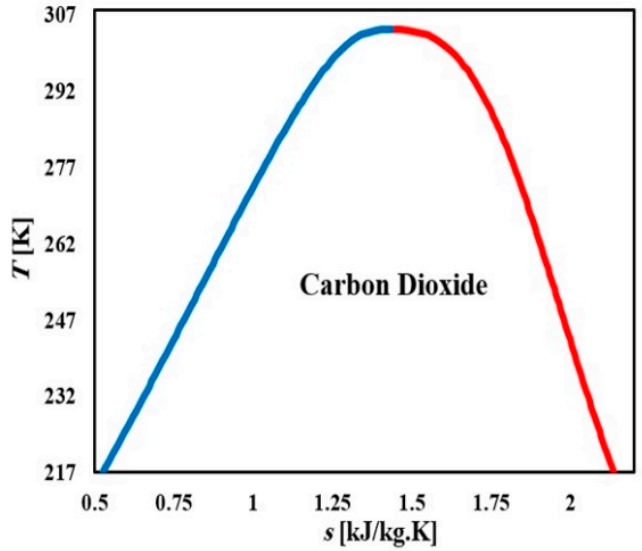

(b)

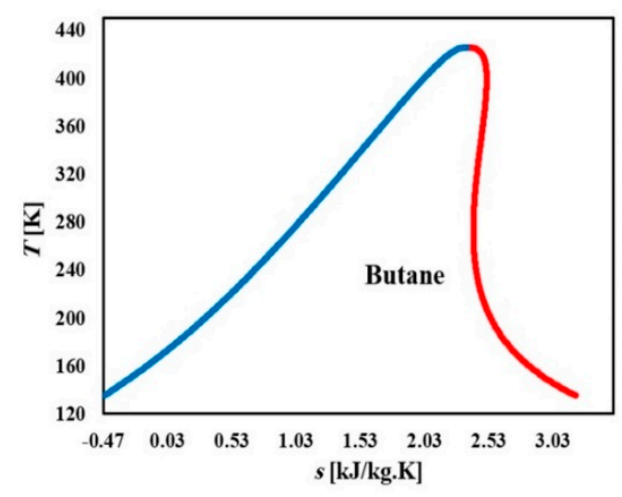

(d)

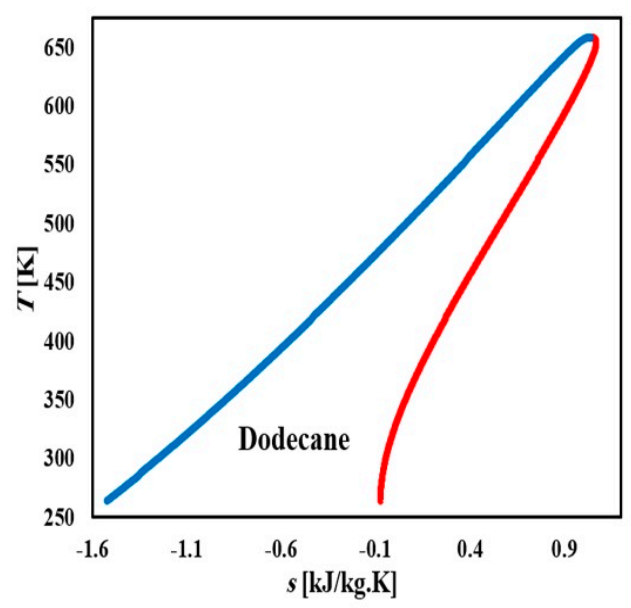

(f)

Figure 2. Temperature dependencies of ORC (or other Rankine-like) and TFC cycles for various working fluids (Water (a), $\mathrm{CO}_{2}(\mathbf{b}), \mathrm{R} 116(\mathbf{c})$, Butane (d), Neopentane (e), Dodecane (f)); see text for further explanation. 
The efficiency values for various maximal and minimal temperature pairs were calculated with MATLAB software; working fluid data were taken from the NIST Chemistry Webbook [25]. On the schematic T-s diagram of a wet working fluid, the blue part represents the saturated liquid state, and the red part represents the saturated vapor state.

The efficiency calculation for ORC and TFC were done in the manner shown in Figure 3. First, a minimal cycle temperature was chosen (represented by the light green line) and used as condenser temperature. For this given condenser temperature, various maximal temperature values were chosen, up to the critical temperature. For each calculation, the temperature interval between the condenser and maximal temperature was divided into 500 equal part; efficiency values were calculated for all of these 500 points, using them as maximal cycle temperatures. Some of them are shown in Figure 3a, corresponding to the evaporation plateau (ORC) and on Figure $3 b$, corresponding to the topmost point of the "triangle" (TFC). In this way, the efficiency of the first cycle (1-2-3-4-1) were calculated. After finishing one run, a new minimal temperature was chosen slightly above the previous one (by shifting the green base-line a bit higher), and the calculation was repeated for the cycle (1-2a-3a-4a-1) up to the last one, just one step below the critical temperature, to cycle (1-2d-3d-4d-1). In this way, separate curves representing the Tmax dependence of efficiencies were obtained for different condenser temperature.

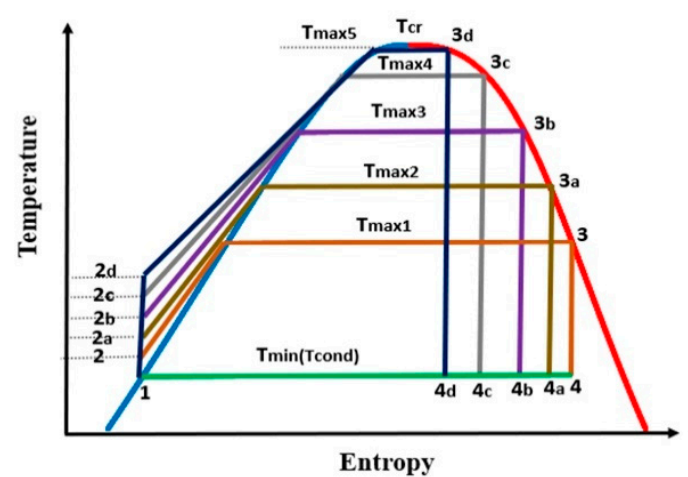

(a)

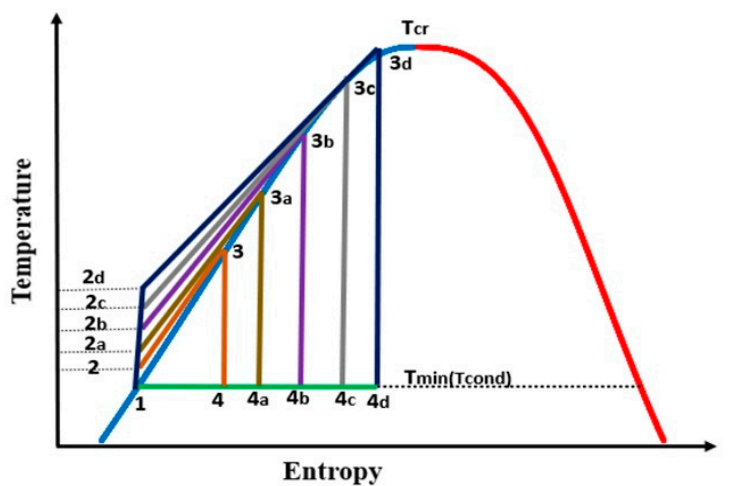

(b)

Figure 3. Steps of the efficiency-calculation for ORC (a) and TFC (b) cycles.

Concerning efficiency, the actual values were calculated by using the following equation:

$$
\eta=\frac{Q_{\text {in }}-Q_{\text {out }}}{Q_{\text {in }}}
$$

where (concerning the isobaric heat exchanges) the heat added to the system is $Q_{i n}=h_{3}-h_{2}$ and the heat taken from the system is $Q_{o u t}=h_{4}-h_{1}$; numerical enthalpy values were taken from the NIST Chemistry Webbook [25] as a function of temperature and entropy.

\section{Results}

On Figure 4, one can see two efficiency lines for $\mathrm{CO}_{2}$; upper one (blue) represents the efficiency of the Rankine-like subcritical $\mathrm{CO}_{2}$ power cycle, while the lower one (green) represents its TFC counterpart. The minimal temperature (condenser temperature) were chosen as the triple-point temperature of the $\mathrm{CO}_{2}(216.6 \mathrm{~K})$, and the efficiencies were calculated up to the critical point (304.13 K). On Figure 4, the whole curve can be seen, while on Figure $4 \mathrm{~b}$, the high-temperature region can be seen, showing the well-developed maximum on the upper curve, 4 degrees below the critical point. 


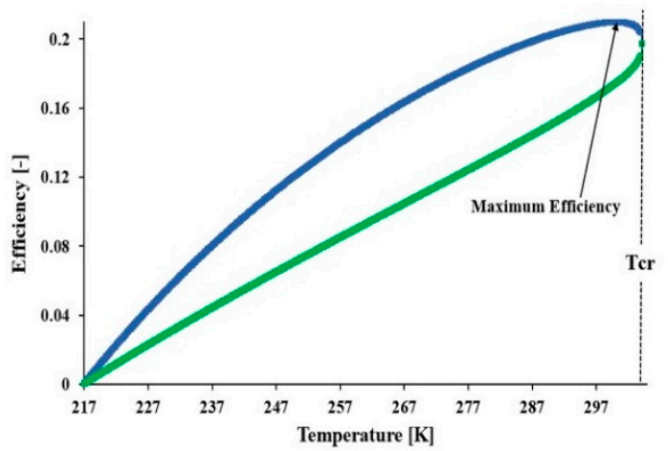

(a)

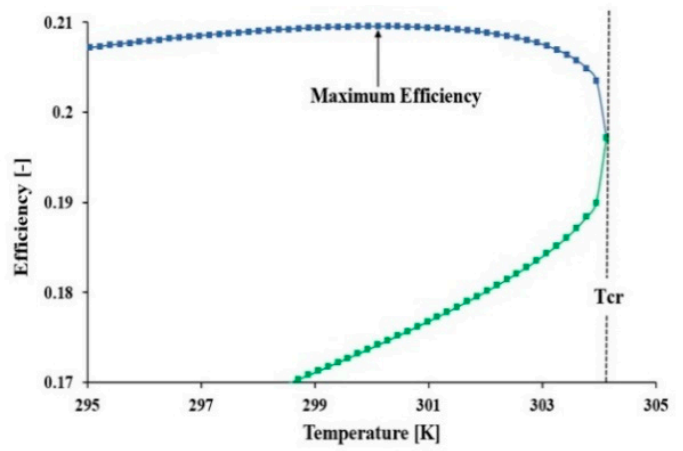

(b)

Figure 4. The efficiency of a $\mathrm{CO}_{2}$ Rankine-like Power Cycle (upper lines) and Trilateral Flash Cycle (lower lines) with fixed condenser temperature $(216.6 \mathrm{~K})$ and with increasing evaporator temperature. (a) full temperature range; (b) the vicinity of the critical temperature.

On Figure 5, a set of these curves (approximately 20 for each material) can be seen for all materials listed in Table 1. The first sign of this maximum was found earlier in butane [26]. For water (5a) and carbon dioxide (5b) (two typical wet working fluids), the maxima on all ORC curves are very characteristic. For $\mathrm{CO}_{2}$, red dots are marking these maxima for better visualization; these points are omitted for the other materials to avoid them to distort the shape of the curves. The increase of the maximal cycle temperature (with a fixed minimal cycle temperature) causes an increase of the efficiency for both cycles (the Rankine and the flash-types, represented by the upper and lower curves). Reaching higher maximal temperatures, the speed of this increase will be lower and lower, and finally a maximum, then a decreasing part can be seen. It means that with a fixed condenser temperature, the increase of maximal temperature has an initially positive effect of the first-law efficiency, but when it is reaching the vicinity of the critical point, this positive effect disappears, and the further increase would be contra-productive. On the contrary, for the TFC-like cycles, the increase holds up to the critical point, i.e., having a TFC-like cycle with a wet working fluid, the increase of maximal cycle temperature always increases the efficiency. In this way, the two curves are forming an elongated rain-drop form; the pointed end is located at the condenser temperature (with $\eta=0$ ). Increasing the condenser temperature, one can obtain smaller efficiencies; both the upper and lower curves are shifting down, while the location of the maximum (with smaller and smaller value) shift closer to the critical temperature (see the location of the red dots for $\mathrm{CO}_{2}$ ), but always remains below that value. The rain-drop shape formed by the upper and lower curves remains, but it will be smaller and smaller. For the sake of the better visibility, the location of the maxima are marked by red dots for the $\mathrm{CO}_{2}$ curves (Figure $5 b$ ); its movement can be clearly seen.

Hexafluoroethane (R116) is originally an almost isentropic working fluid. The T-s diagram has an inverse S-shaped saturated vapor part, which is very narrow, i.e., the entropy value for the vapor phase (except in the vicinity of the critical temperature) is almost constant. In the novel classification system, it is a type ACNMZ. From the mapping of various working fluid types [21], this is an almost wet isentropic fluid. The point $\mathrm{M}$ located at $253.5 \mathrm{~K}$, i.e., choosing condenser temperature above this value, R116 acts like a wet working fluid. Point $\mathrm{N}$ is located at $220.9 \mathrm{~K}$, i.e., choosing condenser temperature between $220.9 \mathrm{~K}$ and $253.5 \mathrm{~K}$, this fluid can mimic a moderately dry working fluid. Efficiency-curves can be seen in Figure $5 \mathrm{c}$. The curves are very similar to the ones seen for water and $\mathrm{CO}_{2}$; the maxima are still well-developed (although these points are closer to the critical point than for the two wet ones), and the joint ORC-TFC curves are also raindrop-shaped. For this material, one can also say that increasing the maximal cycle temperature has a positive effect on the first-law efficiency, but in the vicinity of the critical point, this effect disappears and changes to negative. 


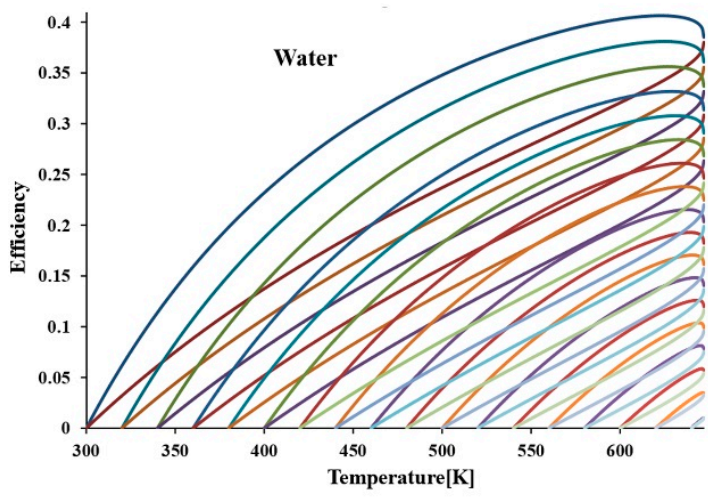

(a)

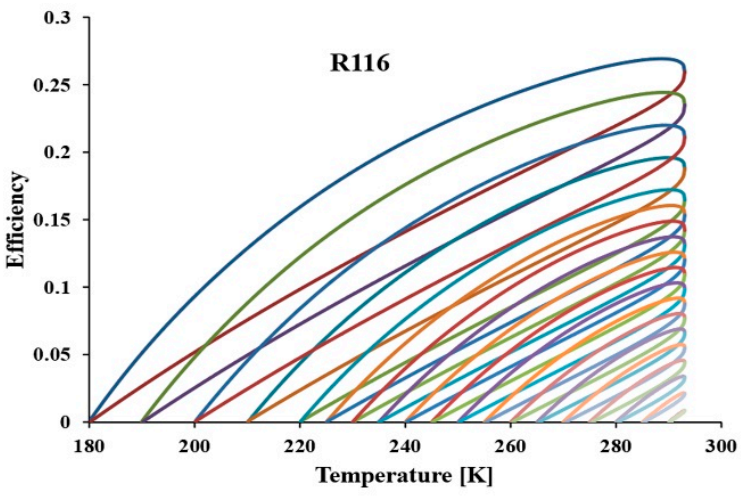

(c)

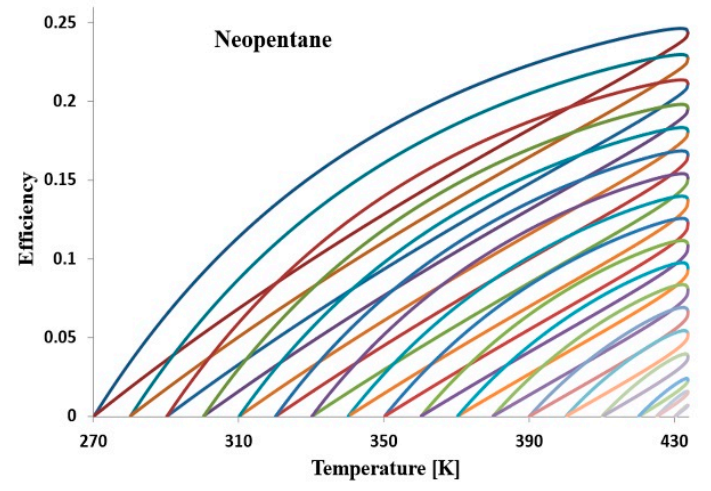

(e)

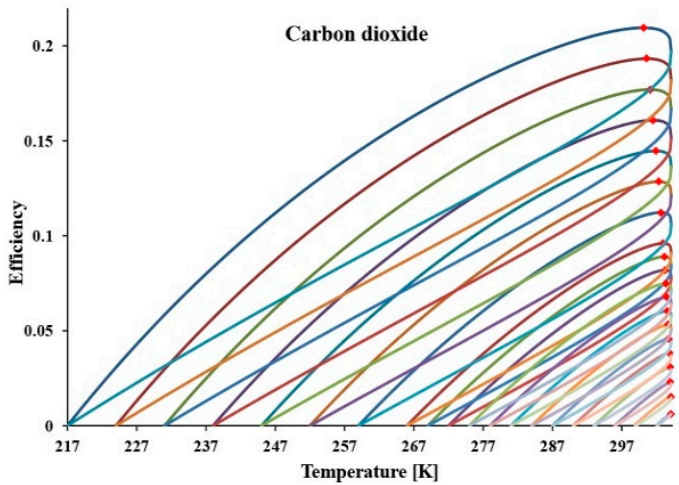

(b)

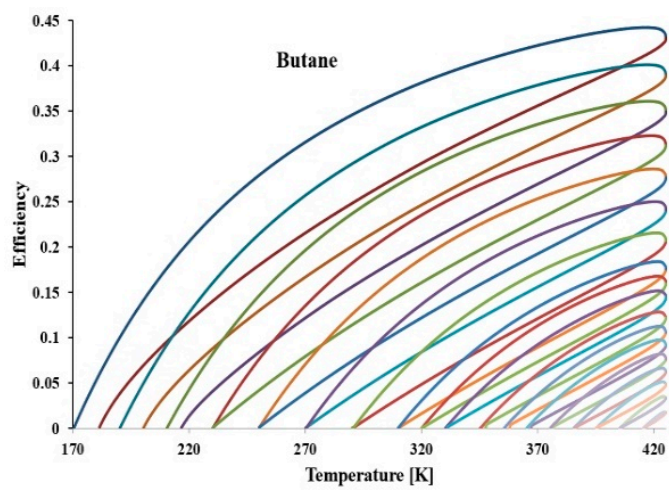

(d)

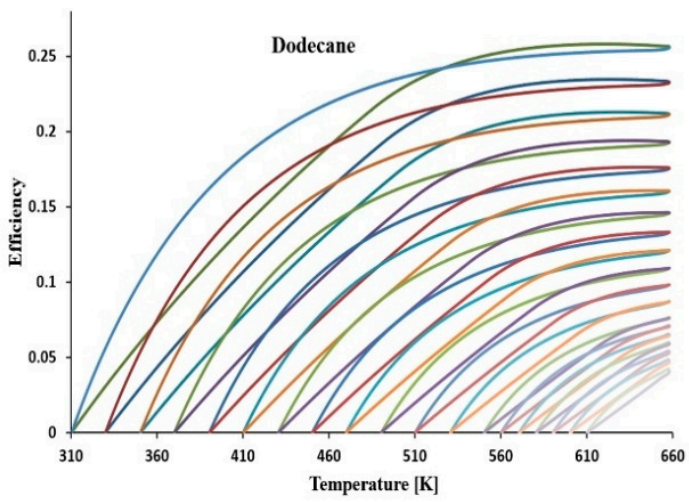

(f)

Figure 5. Temperature dependencies of ORC (or other Rankine-like cycles) and TFC cycles for various working fluids (Water (a), $\mathrm{CO}_{2}$ (b), R116 (c), Butane (d), Neopentane (e), Dodecane (f)); see text for further explanation.

Butane is a moderately dry working fluid; it means that although the T-s diagram is not bell-shaped, but tilted, the titling is not very strong. ORC-TFC efficiency curves can be seen in Figure 5d. Although maxima can be still noticed, they are remarkably less developed than for the previous three materials.

For neopentane (Figure 5e), which has a more tilted T-s diagram that butane (i.e., it is drier), the rain-drop shape is already very distorted, and even the flat end is almost pointed (although the transition from ORC to TFC is still smooth). Very small, nearly diminished maxima can be seen, located very close to the critical temperature. In this case, one can assume, that increase of the maximal cycle temperature is always good or almost neutral for the efficiency. For the uppermost curve, the location of the maximum is only $1.97 \mathrm{~K}$ from the critical temperature, and even above this point, the decrease of efficiency is very small, so, one can consider it almost temperature-independent. 
Finally, a very dry (on T-s diagram, represented by a very tilted $T$-s curve) material, dodecane (Figure 5f) were analyzed. One can see, that at low evaporation temperatures, ORC efficiency exceed TFC one, as it happened before, but increasing this temperature further, the difference will be smaller and smaller, and at a given temperature (which depend on the condenser temperature, i.e., different for each pair of curves) it disappears at the crossing of the two curves. Passing that crossing temperature, the efficiency of TFC is higher than for ORC; finally, they will join smoothly at the critical point. In this case, the efficiencies of TFC can form a shallow maximum, close to the critical curve, while the efficiency of ORC increases continuously. Therefore one can conclude, that by using very dry working fluid for an ideal (strictly isobaric heat exchanges and strictly isentropic expansion/compression) basic-layout (recuperator and superheater-free) ORC-system, the increase of maximal cycle temperature can always increase cycle efficiency, even in the vicinity of the critical temperature; additionally, small maxima might be expected for the efficiency of TFC.

3-D version of these lines for water (Figure 6a-d) and butane (Figure 6e-h) are also shown Rotating animated gif version of these figures are provided as Supplementary Material. The distortion of the initial rain-drop shape can be clearly seen.

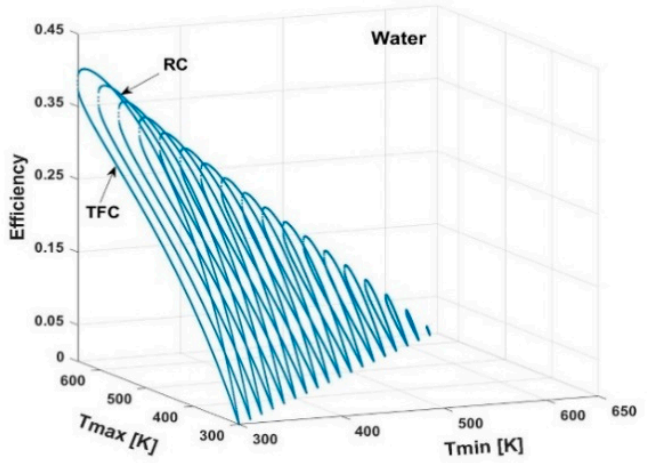

(a)

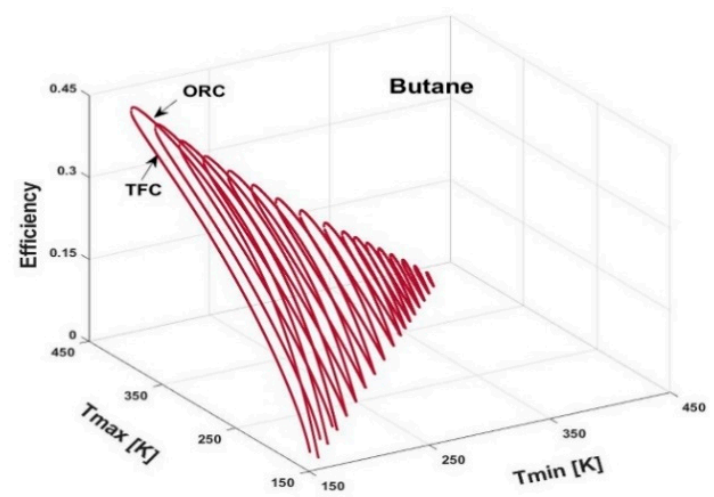

(e)

Figure 6. Cont. 


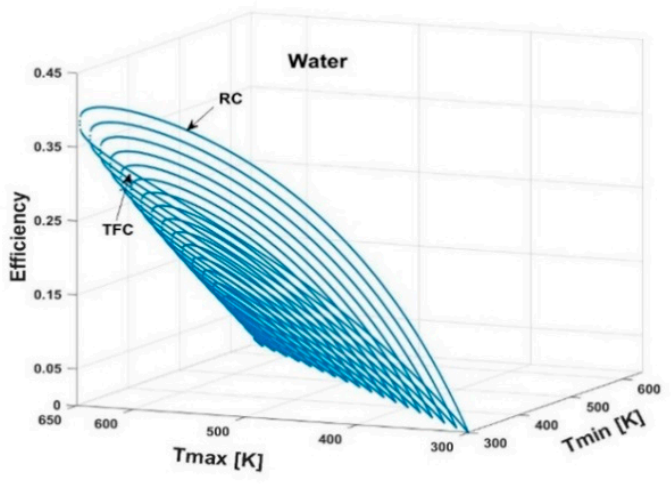

(b)

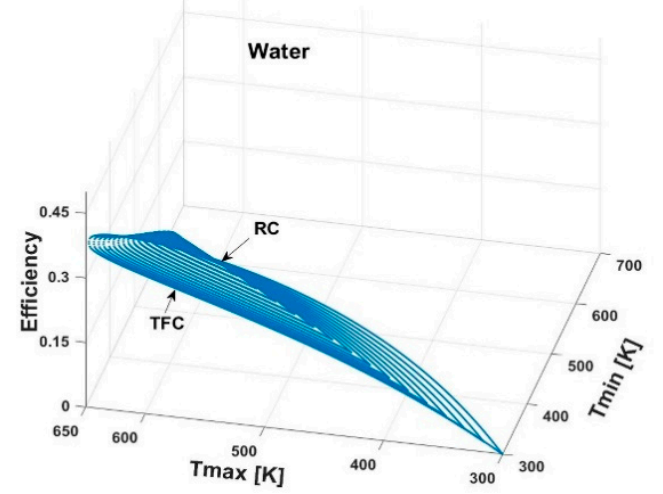

(c)

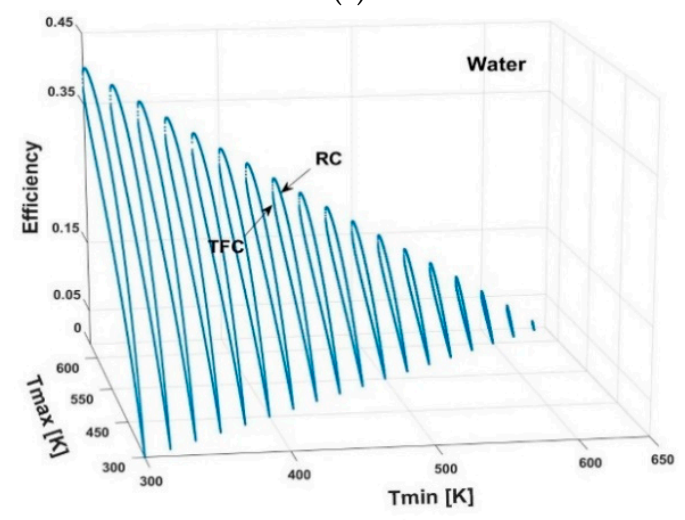

(d)

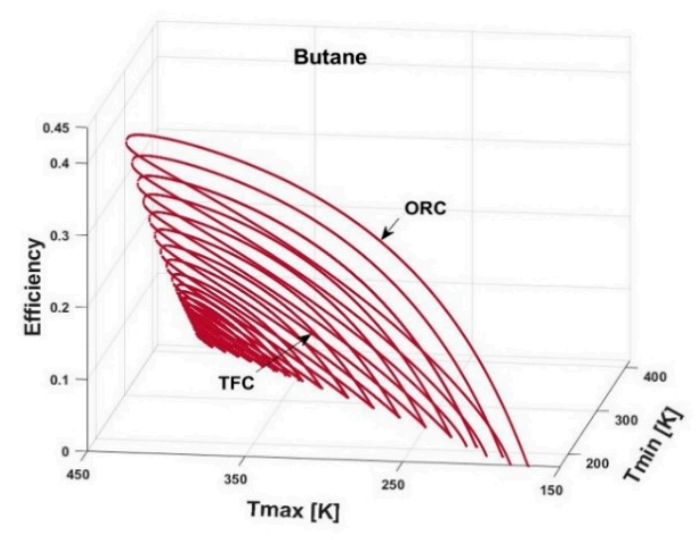

(f)

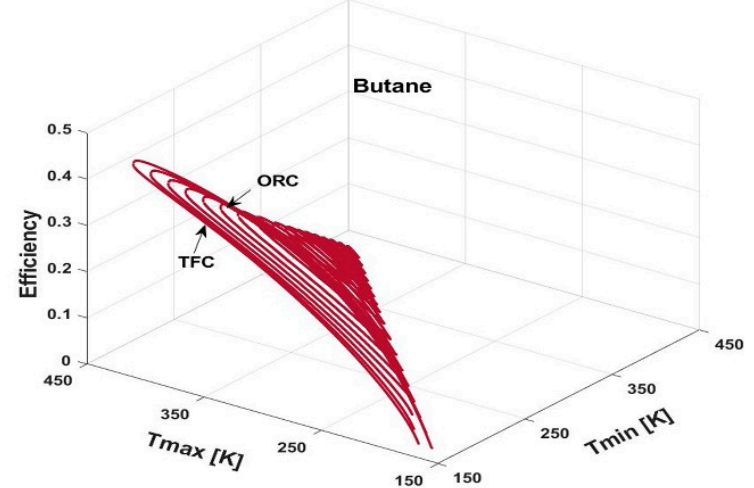

(g)

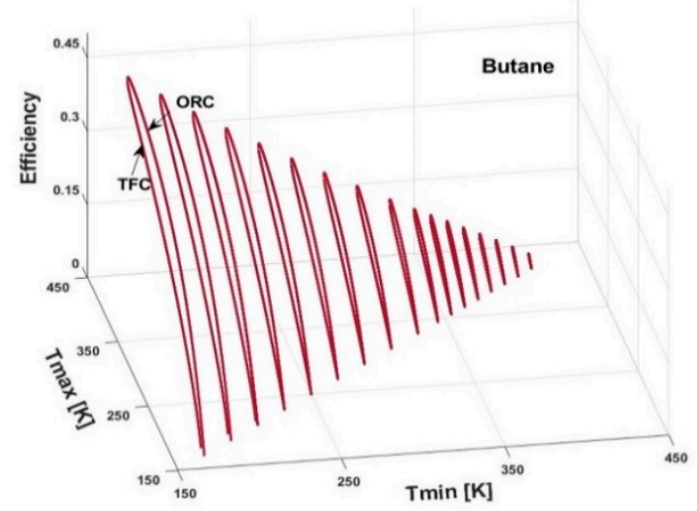

(h)

Figure 6. 3-D representation of the efficiency-curves for water $(\mathbf{a}-\mathbf{d})$ and butane $(\mathbf{e}-\mathbf{h})$. Rotating version of these figures is provided in the Supplementary Material as Figure S1a (water) and Figure S1b (butane) in animated gif-format.

\section{Theoretical Reason for the Existence and Disappearance of the Maximum}

A simple geometrical explanation can be given for the appearance of efficiency maximum in wet working fluids. It can be seen in Figure 7 that the ORC or any other Rankinelike cycle (steam Rankine or $\mathrm{CO}_{2}$ power cycles) can be seen as a superposition of a TFC-like and a Carnot-like cycles, although this superposition is not simply a summing of efficiencies. The TFC-like part is the one at lower entropies, resembling a triangle, while the Carnot-part (located on higher entropies) is the rectangular one. Considering that the Carnot-cycle is the best potential cycle between the given maximal and minimal temperature, adding it to a TFC, the Carnot-like part always has a positive contribution to efficiency. In this way, for wet working fluids, $\eta_{\mathrm{ORC}}$ is always bigger, than $\eta_{\mathrm{TFC}}$. One can see, that by increasing the maximal cycle temperature (compare Figure 7a,b), the rectangular part will be smaller 
and smaller, almost diminishing in the vicinity of the critical temperature (Figure 7c), and when $T_{\max }=T_{\mathrm{cr}}$, it disappears, and the TFC and ORC cycles will be indistinguishable. Therefore, at $T_{\max }=T_{\mathrm{cr}}, \eta_{\mathrm{ORC}}=\eta_{\mathrm{TFC}}$, and the transition will be smooth and continuous.

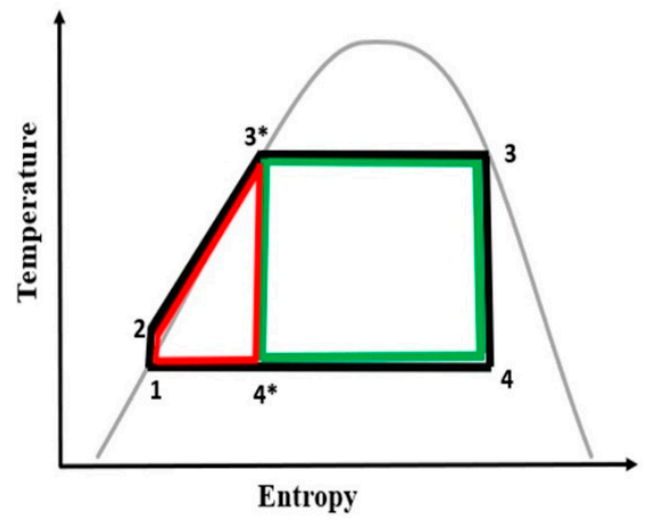

(a)

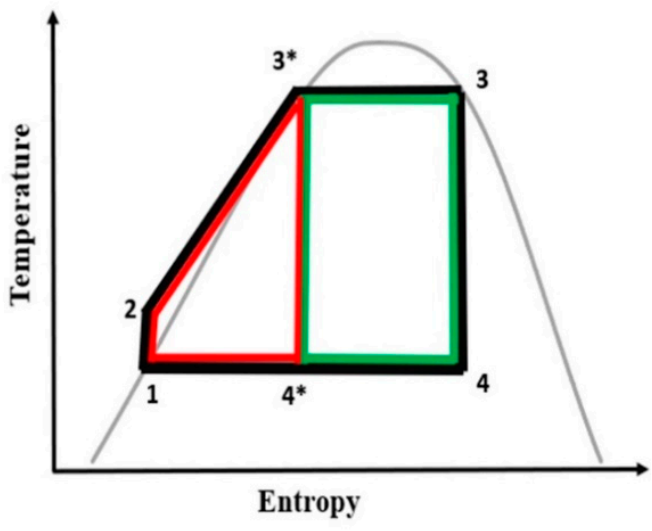

(b)

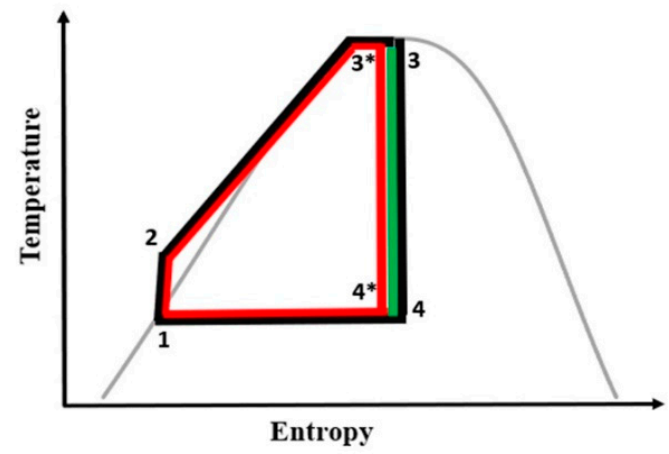

(c)

Figure 7. Rankine-cycle (black) in a wet working fluid as a superposition (but not the simple sum) of a TFC-like (red) and Carnot-like (green) parts with same condenser temperature with a medium (a) high (b) and almost critical (c) evaporator temperature.

This smoothness and continuity affect the shape of the efficiency-temperature curve. Previously we used $T-\eta$ curves, here, for a short time, the inverse should be used, the $\eta-T$ one (see Figure 8). Being $\eta_{\mathrm{ORC}}>\eta_{\mathrm{TFC}}$ for wet working fluids at every temperature, except $T_{\mathrm{cr}}$, the low-entropy part (left side) represents the TFC, and the high-entropy part (right side) represents the ORC. In this representation, the two curves have to have end-points at the maximal temperature $\left(T_{\mathrm{cr}}\right)$. Continuity causes the equality of the two efficiencies at $T_{\mathrm{cr}}$, i.e., the two curves have to join in this point, i.e., instead of two end-points, they will have one common end-point. Due to the smoothness, the derivative $\mathrm{d} T / \mathrm{d} \eta$ will be zero in this point; therefore, this will be a smooth maximum with positive $\mathrm{d} T / \mathrm{d} \eta$ on the left side and negative $\mathrm{d} T / \mathrm{d} \eta$ on the right one. It should be recalled, that the left side represents the efficiency of ORC; being $d T / d \eta$ positive on the original, $T-\eta$ representation, this part would show negative slope. Therefore, in the vicinity of $T_{\mathrm{cr}}$, the smooth and continuous transition between ORC and TFC cycles would cause a part, where efficiency decreases with increasing temperature. Contrary, at lower temperatures, one can see an increasing part; this can be possible only by assuming a maximum on the $\eta(T)$ curve of the ORC.

One should remember that a strong point of this reasoning was to handle ORC as a superposition of a TFC-like and a Carnot-like part. While this is true for the wet working fluid (Figure 9a), in a dry one, a third part, resembling a distorted, upside-down, inverse TFC, can also be defined, see Figure $9 \mathrm{~b}-\mathrm{d}$. This part is also less efficient, that a Carnot-like one. Increasing the "dryness", i.e., having more and more tilted T-s diagrams and going 
from ACZM to AZCM class [23], the weight of the middle Carnot-like part will be smaller and smaller. Finally, this part can completely disappear.

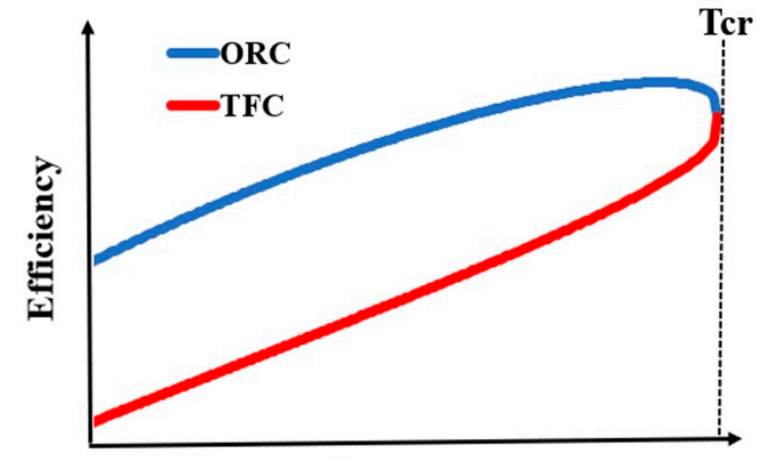

Temperature

(a)

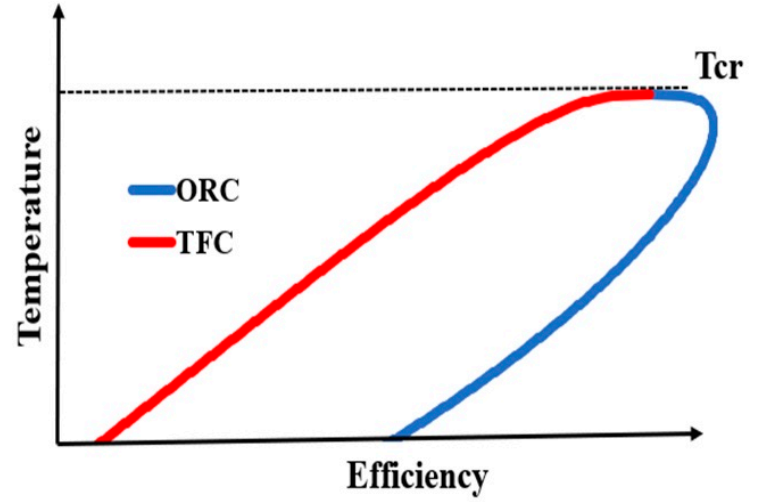

(b)

Figure 8. Schematic temperature-efficiency (a) and its inverse, efficiency-temperature (b) curves of an ORC-like and TFC-like system, explaining the necessity of the maximum. Further explanation can be found in the text.

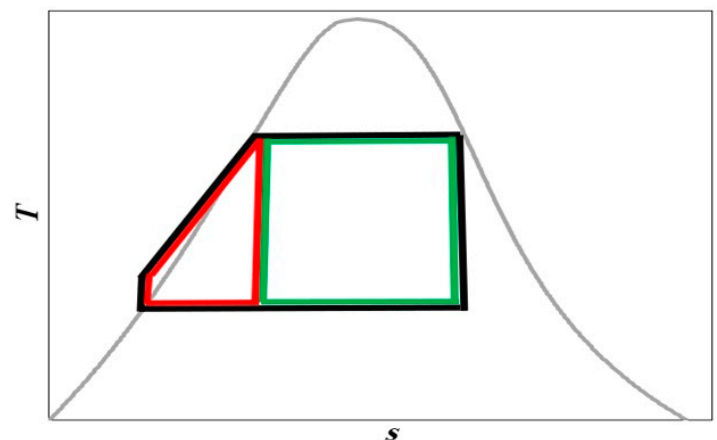

(a)

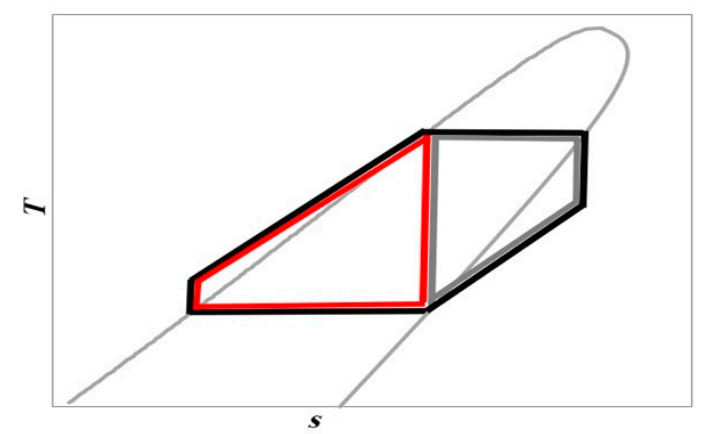

(c)

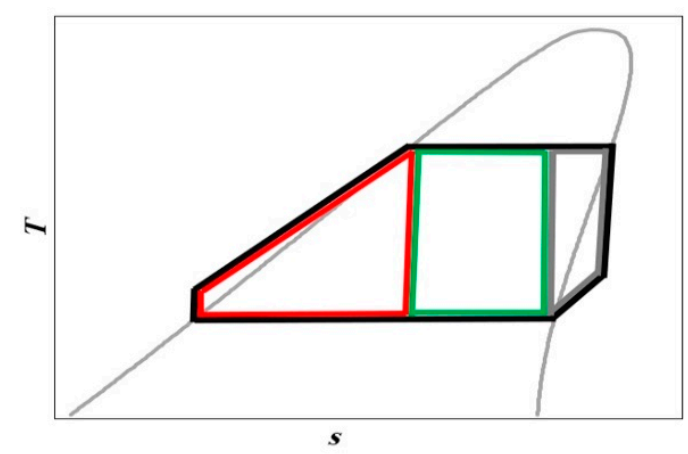

(b)

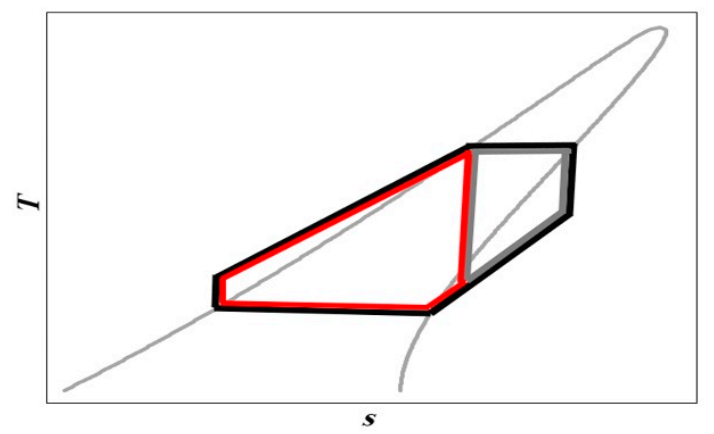

(d)

Figure 9. Different structure of an ORC cycle (black) with a wet, moderately dry, a medium dry and a very dry working fluid, divided into TFC-like (red), Carnot-like (green) and inverse TFC-like (grey) sub-cycles. The diminishment of the Carnot-like part (green) with increasing dryness can be clearly seen, as well as the loss of triangularity of TFC (red) and the appearance of the inverse TFC-like type (grey). The four figures are representing wet (a), moderately dry (b), medium dry (c) and very dry (d) working fluids.

This process can be seen in detail in Figure 9a-c. With wet working fluid (a), the ORC can be seen as a superposition of an almost perfectly triangular TFC (red) and an ideal Carnot (green) cycles, as it has been shown before (Figure 7). For moderately dry one (b), a third part (grey) appears, located at higher entropies, resembling a distorted upsidedown TFC. The appearance of this part makes the Carnot-like part narrower, decreasing 
its superficial contribution to net first-law efficiency. For medium dry one (c) the isobaric, but not isothermal part of the heat removal is already so significant, that the Carnot-like part totally disappears and the cycle will be the superposition of an almost triangular TFClike and an upside-down TFC-like part; because the Carnot-part disappeared, one might expect that net efficiency might be smaller than previously. Finally, with a very dry working fluid, the isobaric heat removal part preceding the condensation will be so huge, that it will reach the initial TFC-like part, cutting down the corner and terminating the "triangularity" (Figure 9d).

Because this transition happens with increasing dryness, one can expect to see the similar process by going from wet (water, $\mathrm{CO}_{2}$ ) through isentropic (R116), moderately dry (butane) and dry (neopentane) working fluids to very dry one (dodecane). Upon this transition, the maximum will be less and less significant, and finally, it can disappear, as it is shown for dodecane.

\section{Discussion and Conclusions}

The increase of the maximal cycle temperature (which is equal to the evaporator temperature in ideal, simple, superheater-free systems) is thought to be a good way to increase the first-law efficiency in simple cycles, like steam Rankine, Organic Rankine and $\mathrm{CO}_{2}$ power cycles, although there are signs, that problems might arise when this increase reaches the vicinity of the critical point. Some of the problems related to the properties of the materials in this region; there are significant density fluctuations, causing strong local anomalies; also, the accuracy of the Equation of States can be reduced in this region $[27,28]$; this can also produce problems during design and in the operational phases. Also, from the technical point of view, high evaporation temperatures, especially for dry working fluids, are also not very favorable $[29,30]$. For example, choosing the evaporation temperature above the temperature of local entropy maximum (the so-called point $\mathrm{M}$ ), expansion starts in the wet region, even in dry fluids; therefore, droplets can be formed during the early stage of the expansion, causing troubles in the special "dry" expanders.

Here, we approached the problem of the increase of maximal cycle temperature from a purely thermodynamic side, by determining its effect (with fixed condenser temperature) on the first-law efficiency. Efficiencies for six different materials with increasing dryness (water, $\mathrm{CO}_{2}, \mathrm{R} 116$, butane, neopentane and dodecane) were evaluated. Although anomalies were expected to be seen for dry working fluids [29,30], surprisingly, a very strange effect was seen for wet working fluids, namely the appearance of an efficiency maximum on the efficiency of the Rankine-like cycle (first seen for butane [26]), located close to the critical temperature. The location of the maximum depends on the condenser temperature as well as on the material. For water, taking the smallest possible condenser temperature (the triple point temperature, $273.16 \mathrm{~K}$ ), the maximum appears $24.6 \mathrm{~K}$ below the critical point, while increasing the condenser temperature, it can approach the critical point. For $\mathrm{CO}_{2}$, R116 and butane, the biggest distance between the location of the maximum from the critical point is in the order of $5-10 \mathrm{~K}\left(8.68 \mathrm{~K}\right.$ for butane, $4.01 \mathrm{~K}$ for $\mathrm{CO}_{2}$ and $4.53 \mathrm{~K}$ for R116); for neopentane, it is $1.97 \mathrm{~K}$, and finally, for dodecane, no maximum can be seen. Concerning reduced temperatures, the biggest distances between the entropy maximum and the critical points are 0.038 (water), $0.013\left(\mathrm{CO}_{2}\right), 0.015$ (R116), 0.020 (butane) and 0.005 (neopentane). Since for most real ORC and RC power plants, the operational temperature is not very near to the critical temperature; therefore, technologically, the existence of the maxima are relevant only for two cases (for water, where the maximal distance between the critical temperature end efficiency maximum is almost $25 \mathrm{~K}$, and for butane, where this difference is almost $9 \mathrm{~K}$ ). For the rest of the presented working fluids, the relevance is rather theoretical than technical. However, one might assume that the temperature differences will be sufficient to have technological importance for some of the not presented wet working fluids.

The presence of these maxima can be clearly shown for several systems by comparing the efficiency of a Rankine-like cycle with a Trilateral Flash-like Cycle (TFC). Some theo- 
retical explanation is also given to see, that for very dry working fluids (like dodecane), the disappearance of this maximum can always be expected for simple, Rankine-like cycles, using only the basic Rankine-layout (pump, liquid heater with evaporator, expander and condenser), without recuperative unit.

The existence of the efficiency maximum for various working fluids is a disproof of the common misconception that the increase of the maximal cycle temperature always has a positive effect on first law efficiency for simple thermodynamic cycles. This disproof is interesting itself, as a strange thermodynamic fact, but also, it can give a new tool for engineers to find optimal cycle temperature for an ORC (or similar) power plant, by designating a temperature range, where a further increase of the evaporation temperature would be contra-productive.

Supplementary Materials: The following are available online at https:/ / www.mdpi.com/1996-1 073/14/2/307/s1, Figure S1a,b: Rotating 3-D representation of the efficiency-curves for water and butane, respectively (in animated gif-format).

Author Contributions: Conceptualization, A.R.I.; analysis: A.M.A., preliminary analysis: L.K., writing: A.R.I., A.M.A. and L.K. All authors have read and agreed to the published version of the manuscript.

Funding: This work was performed in the frame of the FIEK_16-1-2016-0007 project, implemented with the support provided from the National Research, Development and Innovation Fund of Hungary, financed under the FIEK_16 funding scheme. Part of the research reported in this paper and carried out at BME has been supported by the NRDI Fund (TKP2020 NC, Grant No. BME-NC) based on the charter of bolster issued by the NRDI Office under the auspices of the Ministry for Innovation and Technology.

Institutional Review Board Statement: Not applicable.

Informed Consent Statement: Not applicable.

Data Availability Statement: The data that support the findings of this study are available from the corresponding author, [A.R.I.], upon request.

Conflicts of Interest: The authors declare no conflict of interest.

\section{Appendix A. Hybrid Solar Systems}

Renewable energy is environmentally friendly; therefore, it is the most viable future option to dispose of some of the environmental problems. Solar is one of the best renewable energy sources, due to availability and high temperature, wherefore solar thermal power, or concentrated solar power (CSP), is an optimal technology to hybridize with other energy technologies for power generation. The hybrid systems using geothermal and solar energy is one of the options with good potentials. This hybrid technology has a promising future, especially in countries that have abundant solar and geothermal energy. The stand-alone geothermal energy can be classified as medium- or low-enthalpy heat source for power plants, therefore, adding solar energy is the best choice, due to increasing the temperature of a geofluid, which increases the first-law efficiency to convert heat to work (and then to electricity) [15,16,31].

In general, a hybrid system can be categorized based on the kind of used energy, such as merging the solar source with one of the renewable energy sources like geothermal, wind, and biomass (these are the so-called high-renewable hybrids); while merging it with natural gas is called medium-renewable hybrids. Finally, merging it with traditionally fueled Brayton or/and Rankine cycle, the systems are called as a low-renewable hybrid.

Reducing $\mathrm{CO}_{2}$ emission is a crucial factor for the power plant. The lowest $\mathrm{CO}_{2}$ emission is with high-renewable hybrids, and the rate increases with medium-renewable, and it is at its highest rate with the low-renewable hybrids; therefore, the increase of the solar part reduces the $\mathrm{CO}_{2}$ emissions [32]. The efficiency of hybrid systems can be increased by adding thermal energy storage that uses the surplus of solar thermal energy 
at the night time when the temperature is low. Furthermore, the increase of the solar collector field increases the efficiency in all case with or without using thermal energy storage [33]. The second-law efficiency for the hybrid geothermal-solar system is higher than for separate geothermal and solar system at all ambient temperatures [34]. Concerning the increase of maximal temperature above the critical point, the hybrid supercritical ORC (Organic Rankine cycle) outperforms the hybrid subcritical ORC thermodynamically and economically. Therefore, the hybrid supercritical ORC has higher thermal efficiency, and the ability to produce power two to nine times more than a stand-alone subcritical geothermal plant. The geothermal-solar plant is characterized by produce relative constant power during its period operation, concerned to stand-alone solar ones [18,35].

Concerning working fluids, some preliminary result was achieved, showing that that isobutene (also called isobutylene) is a suitable working fluid in the hybrid geothermalsolar system; it has the best performance with the lower GWP, when compared to other working fluids as isobutene, $n$-butane, and 2-butene [36]. The hybrid solar-geothermal is less vulnerable to the seasonal changes in ambient temperatures than stand-alone solar one, as it was observed in an Australian geothermal-solar hybrid system case study, demonstrating that a properly designed hybrid plant can outperform a stand-alone solar thermal plant in terms of the cost of electricity production [37].

As a final issue, the thermal stability of the working fluid and the capacity of the system components might limit the solar superheating in the hybrid geothermal-solar so, to overcome this problem, water can be used as the heat transfer fluid for ORC that operates under $175^{\circ} \mathrm{C}$ [38].

\section{References}

1. Struchtrup, H. Thermodynamics and Energy Conversion; Springer: Berlin/Heidelberg, Germany, 2016.

2. Nag, P.K. Power Plant Engineering, 3rd ed.; Tata McGraw-Hill: New Delhi, India, 2008.

3. Macchi, E.; Astolfi, M. Organic Rankine Cycle (ORC) Power Systems: Technologies and Applications; Elsevier-Woodhead Publishing: Duxford, UK, 2016.

4. Quoilin, S.; Van Den Broek, M.; Declaye, S.; Dewallef, P.; Lemort, V. Technoeconomic survey of Organic Rankine Cycle (ORC) systems. Renew. Sustain. Energy Rev. 2013, 22, 168-186. [CrossRef]

5. Morrison, G. The shape of the temperature-entropy saturation boundary. Int. J. Refrig. 1994, 17, 494. [CrossRef]

6. Chen, H.; Goswami, D.Y.; Stefanakos, E.K. A review of thermodynamic cycles and working fluids for the conversion of low-grade heat. Renew. Sustain. Energy Rev. 2010, 14, 3059-3067. [CrossRef]

7. Garrido, J.M.; Quinteros-Lama, H.; Mejía, A.; Wisniak, J.; Segura, H. A rigorous approach for predicting the slope and curvature of the temperature-entropy saturation boundary of pure fluids. Energy 2012, 45, 888-899. [CrossRef]

8. White, J.A.; Velasco, S.A. Simple Semiempirical Method for Predicting the Temperature-Entropy Saturation Curve of Pure Fluids. Ind. Eng. Chem. Res. 2019, 58, 1038-1043. [CrossRef]

9. Bao, J.; Zhao, L. A review of working fluid and expander selections for Organic Rankine Cycle. Renew. Sustain. Energy Rev. 2013, 24, 325-342. [CrossRef]

10. Kolasiński, P. The Method of the Working Fluid Selection for Organic Rankine Cycle (ORC) Systems Employing Volumetric Expanders. Energies 2020, 13, 573. [CrossRef]

11. Groniewsky, A.; Wagner, C. Investigation of the Effect of the Regenerative Heat Exchanger on the Performance of Organic Rankine Cycles Using Perturbed Chain-Statistical Associating Fluid Theory Equation of State. Ind. Eng. Chem. Res. 2020, 59, 19643-19656. [CrossRef]

12. Groniewsky, A.; Györke, G.; Imre, A.R. Description of wet-to-dry transition in model ORC working fluids. Appl. Therm. Eng. 2017, 125, 963-971. [CrossRef]

13. Tóth, A.N. The Geothermal Atlas of Hungary, 1st ed.; Hungarian Energy and Public Utility Regulatory Authority: Budapest, Hungary, 2016.

14. Maali, R.; Khir, T. Performance analysis of different ORC power plant configurations using solar and geothermal heat sources. Int. J. Green Energy 2020, 17, 349-362. [CrossRef]

15. Mohammadi, K.; Khanmohammadi, S.; Khorasanizadeh, H.; Powell, K. A comprehensive review of solar only and hybrid solar driven multigeneration systems: Classifications, benefits, design and prospective. Appl. Energy 2020, 268, 114940. [CrossRef]

16. Li, K.; Liu, C.; Jiang, S.; Chen, Y. Review on hybrid geothermal and solar power systems. J. Clean. Prod. 2020, $250,119481$. [CrossRef]

17. Heberle, F.; Hofer, M.; Brüggemann, D. A Retrofit for Geothermal Organic Rankine Cycles based on Concentrated Solar Thermal Systems. Energy Procedia 2018, 129, 692-699. [CrossRef] 
18. Zhou, C. Hybridization of solar and geothermal energy in both subcritical and supercritical Organic Rankine Cycles. Energy Convers. Manag. 2014, 81, 72-82. [CrossRef]

19. Hung, T.-C. Waste heat recovery of organic Rankine cycle using dry fluids. Energy Convers. Manag. 2001, 42, 539-553. [CrossRef]

20. Sadaghiani, M.S.; Ahmadi, M.H.; Mehrpooya, M.; Pourfayaz, P.; Feidt, M. Process development and thermodynamic analysis of a novel power generation plant driven by geothermal energy with liquefied natural gas as its heat sink. Appl. Therm. Eng. 2018, 133, 645-658. [CrossRef]

21. Imre, A.R.; Kustán, R.; Groniewsky, A. Mapping of the Temperature-Entropy Diagrams of van der Waals Fluids. Energies 2020, 13, 1519. [CrossRef]

22. Daniarta, S.; Imre, A.R. Cold Energy Utilization in LNG Regasification System Using Organic Rankine Cycle and Trilateral Flash Cycle. Period. Polytech. Mech. Eng. 2020, 64, 342-349. [CrossRef]

23. Györke, G.; Deiters, U.K.; Groniewsky, A.; Lassu, I.; Imre, A.R. Novel Classification of Pure Working Fluids for Organic Rankine Cycle. Energy 2018, 145, 288-300. [CrossRef]

24. Imre, A.R.; Kustán, R.; Groniewsky, A. Thermodynamic Selection of the Optimal Working Fluid for Organic Rankine Cycles. Energies 2019, 12, 2028. [CrossRef]

25. NIST Chemistry WebBook. NIST Standard Reference Database Number 69. 2018. Available online: http://webbook.nist.gov/ chemistry/ (accessed on 1 February 2020).

26. Kondor, L. Efficiency-increase of low-temperature. Organic Rankine Cycles with Solar Collectors-Thermodynamic and Economic Study. Master's Thesis, Budapest University of Technology and Economics, Budapest, Hungary, June 2020.

27. Kraska, T.; Deiters, U.K. An Equation of State for Pure Fluids Describing the Critical Region. Int. J. Thermophys. 1994, 15, $261-281$. [CrossRef]

28. Dua, G.; Hu, J. An equation of state for accurate thermodynamic modeling of water and carbon dioxide from triple points to 647 K and 100-200 MPa. Int. J. Greenh. Gas Control 2016, 49, 94-107. [CrossRef]

29. Zhang, X.; Zhang, C.; He, M.; Wang, J. Selection and Evaluation of Dry and Isentropic Organic Working Fluids Used in Organic Rankine Cycle Based on the Turning Point on Their Saturated Vapor Curves. J. Therm. Sci. 2019, 28, 643-658. [CrossRef]

30. Zhang, X.; Zhang, Y.; Wang, J. New classification of dry and isentropic working fluids and a method used to determine their optimal or worst condensation temperature used in Organic Rankine Cycle. Energy 2020, 201, 117722. [CrossRef]

31. Powell, K.M.; Rashid, K.; Ellingwood, K.; Tuttle, J.; Iverson, B.D. Hybrid concentrated solar thermal power systems: A review. Renew. Sustain. Energy Rev. 2017, 80, 215-237. [CrossRef]

32. Pramanik, S.; Ravikrishna, R.V. A review of concentrated solar power hybrid technologies. Appl. Therm. Eng. 2017, 127, 602-637. [CrossRef]

33. Bassetti, M.C.; Consoli, D.; Manente, G.; Lazzaretto, A. Design and off-design models of a hybrid geothermal-solar power plant enhanced by a thermal storage. Renew. Energy 2018, 128, 460-472. [CrossRef]

34. Ghasemi, H.; Sheu, E.; Tizzanini, A.; Paci, M.; Mitsos, A. Hybrid solar-Geothermal power generation: Optimal retrofitting Appl. Energy 2014, 131, 158-170. [CrossRef]

35. Bokelman, B.; Michaelides, E.; Michaelides, D. A Geothermal-Solar Hybrid Power Plant with Thermal Energy Storage. Energies 2020, 13, 1018. [CrossRef]

36. Keshvarparast, A.; Ajarostaghi, S.M.A.; Delavar, M.A. Thermodynamic analysis the performance of hybrid solar-geothermal power plant equipped with air-cooled condenser. Appl. Therm. Eng. 2020, 172, 115160. [CrossRef]

37. Zhou, C.; Doroodchi, E.; Moghtaderi, B. An in-depth assessment of hybrid solar-Geothermal power generation. Energy Convers. Manag. 2013, 74, 88-101. [CrossRef]

38. Heberle, F.; Hofer, M.; Ürlings, N.; Schr, H.; Anderlohr, T.; Brüggemann, D. Techno-economic analysis of a solar thermal retrofit for an air-cooled geothermal Organic Rankine Cycle power plant. Renew. Energy 2017, 113, 494-502. [CrossRef] 